\title{
Aspergillosis in a Patient Receiving Temozolomide for the Treatment of Glioblastoma
}

\author{
Rodrigo Ramella Munhoz ${ }^{\mathrm{a}} \quad$ Andrea Arvai Pereira Picarelli ${ }^{\mathrm{b}}$ \\ Cristina Aparecida Troques Mitteldorf ${ }^{c}$ Olavo Feher $^{a}$ \\ ${ }^{a}$ Centro de Oncologia, ${ }^{b}$ Núcleo Avançado do Tórax, and ' Laboratory of Surgical and \\ Molecular Pathology, Hospital Sírio Libanês, São Paulo, Brazil
}

\section{Key Words}

Pulmonary aspergillosis $\cdot$ Temozolomide $\cdot$ Glioblastoma $\cdot$ Opportunistic infections

\begin{abstract}
Leukopenia and selective CD4+ lymphopenia represent major adverse events associated with the use of temozolomide (TMZ), an oral alkylating agent incorporated in the treatment of glioblastoma (GBM). The increased risk of opportunistic infections, including those caused by Pneumocystis jiroveci and cytomegalovirus, has been previously described in the literature. Here we report the case, the first to our knowledge, of a patient with pulmonary invasive aspergillosis immediately after the completion of chemoradiation with TMZ for GBM. Diagnosis was confirmed through a CT-guided lung biopsy, and the patient had excellent response to systemic voriconazole. This case illustrates that TMZ can be associated with severe opportunistic infections, presumably associated with T lymphocyte immune dysfunction, and patients exposed to this agent should be carefully monitored.
\end{abstract}

(C) 2013 S. Karger AG, Basel

\section{Introduction}

Glioblastoma (GBM) is the most frequent primary central nervous system malignancy, with a peak incidence between 75 and 84 years of age $[1,2]$. Temozolomide (TMZ) given concurrently with radiation therapy followed by the same agent as monotherapy for 6 months to 1 year is the current standard of care for patients with newly diagnosed GBM [3, 4].

Rodrigo Ramella Munhoz, MD

Centro de Oncologia, Hospital Sírio Libanês, 2nd floor

Rua Dona Adma Jafet, 91, Bela Vista

São Paulo, SP 01308-050 (Brazil)

E-Mailmunhozrs@gmail.com 
Munhoz et al.: Aspergillosis in a Patient Receiving Temozolomide for the Treatment of Glioblastoma

Although grade 3-4 absolute leukopenia and neutropenia rarely occur, almost $80 \%$ of patients receiving TMZ are at risk of severe lymphopenia [3-5]. Most importantly, impaired cellular immune response resulting from selective CD4+ T-cell depletion has been described in patients receiving TMZ for the treatment of different malignancies [6-8]. The occurrence of opportunistic infections during treatment with TMZ is a well-documented complication, especially those caused by Pneumocystis jiroveci, which led the US Food and Drug Administration (FDA) to recommend the use of routine prophylaxis [9]. Other pathogens, including cytomegalovirus and varicella zoster virus, have also been anecdotally reported in the context of TMZ use $[10,11]$.

Here we report the case, the first to our knowledge, of invasive pulmonary aspergillosis in a patient receiving TMZ for treatment of newly diagnosed GBM.

\section{Case Description}

A 78-year-old woman was diagnosed 4 months before admission with a right frontal GBM with extension to the basal ganglia and temporal lobe. The patient underwent a frontal craniotomy and partial resection of the lesion, followed by hypofractionated radiation therapy (16 fractions of $250 \mathrm{cGy}$ ) with concomitant daily TMZ $\left(75 \mathrm{mg} / \mathrm{m}^{2}\right)$. During the concurrent phase, she developed grade 1 lymphopenia and grade 2 nausea, but the treatment was otherwise well tolerated. Two days after completing chemoradiation, the patient was admitted for dyspnea and asymmetrical lower extremity edema. At admission, she denied cough, sputum, hemoptysis or thoracic pain. She reported no fever, chills, sweats or weight loss. Neurologic symptoms were stable since her last assessment. She had a history of hypertension and controlled type 2 diabetes. Medications included omeprazole, amlodipine, metformin, gliclazide, sitagliptin and prophylactic sulfamethoxazoletrimethoprim. She was also taking dexamethasone $4 \mathrm{mg} /$ day on a tapering scale.

Upon physical examination, she had normal vital signs, with a respiratory rate of 18 breaths/min and oxygen saturation of $95 \%$ on room air. She had asymmetric edema of the lower limbs and, on auscultation, fine respiratory crackles bilaterally. Electrolytes, liver enzymes and renal function were normal. Blood counts were: hemoglobin $11.9 \mathrm{~g} / \mathrm{dl}$, platelets $67,000 / \mathrm{mm}^{3}$ and leukocytes $7,030 / \mathrm{mm}^{3}$, with an absolute lymphocyte count of $800 / \mathrm{mm}^{3}$. A venous duplex-scan of the lower extremities showed an extensive deep vein thrombosis involving the left common femoral vein. An angiotomography of the thorax diagnosed an acute pulmonary embolism and also revealed scattered lobulated nodules with areas of cavitation, measuring up to $2.7 \mathrm{~cm}$, located predominately in the upper lobes, some with a surrounding ground-glass halo. There was no pleural effusion. Areas of peribronchial infiltrates and centrilobular nodules were also present (fig. 1). Serum galactomannan levels, determined using an enzyme-linked immunosorbent assay, were elevated (optical density index $=6.7$, optical density index $\geq 0.5$ is considered a positive result). Blood samples obtained for culture and Cryptococcus neoformans and Histoplasma antigen assays were negative.

A CT-guided pulmonary biopsy was then performed. Histochemical analysis (Gomori's silver stain) showed septate hyphal elements with acute-angle branching (fig. 2), and culture of the pulmonary tissue was positive for Aspergillus fumigatus. The patient was promptly started on antifungal therapy with voriconazole and was discharged to complete the treatment as an outpatient. Chemotherapy with TMZ was resumed after 4 weeks of antifungal therapy. A control CT scan done approximately 30 days after admission revealed a reduction of the nodular lesions and ground-glass component previously identified (fig. 3). 
Munhoz et al.: Aspergillosis in a Patient Receiving Temozolomide for the Treatment of Glioblastoma

Written informed consent for publication of the clinical details and/or clinical images was obtained from the guardian/relative of the patient.

\section{Discussion}

GBM is the most frequent primary central nervous system malignancy [1, 2]. Surgery followed by concurrent radiation, TMZ and adjuvant TMZ is the current standard of care for patients with newly diagnosed GBM [3, 4]. In this report, we present the case of a 78-yearold female diagnosed with pulmonary aspergillosis shortly after completing concurrent chemoradiation. The patient was treated with voriconazole, which is the drug of choice for the treatment of invasive aspergillosis according to published guidelines [12].

In most patients, TMZ is well tolerated, and major adverse events include nausea, vomiting and myelotoxicity, mostly thrombocytopenia and lymphopenia. Lymphocyte count depletion may result in an increased risk of opportunistic infections, and selective CD4+ Tcell depletion was reported in patients receiving TMZ for the treatment of melanoma and neuroendocrine tumors [7-9]. Albeit uncommon, the association of TMZ with P. jiroveci pneumonia is well described in the literature, and the use of prophylaxis with SMX-TMP is currently recommended by the FDA $[3,6,10]$. Among 64 patients accrued in the phase II trial by Stupp et al. [5], 2 developed $P$. jiroveci pneumonia. In the landmark EORTC/NCIC trial, $3 \%$ of patients receiving radiotherapy plus TMZ had severe infections, but only 2 cases of proven opportunistic infections occurred [3,4]. Scarce reports in the literature also suggest an increased risk of infection by other agents, including cytomegalovirus and varicella zoster virus $[10,11]$.

Pulmonary aspergillosis is an uncommon condition in healthy individuals and occurs most frequently in the setting of immunosuppression, including patients with acquired immunodeficiency syndrome and those undergoing treatment for hematological malignancies associated with prolonged neutropenia and high doses of corticosteroids [13]. Aspergillus is a ubiquitous fungus that is often identified in cultures of respiratory fluids even in the absence of infection. Drugs that result in impaired cellular immune response mechanisms could result in an increased risk of pulmonary aspergillosis. The classic triad of pulmonary aspergillosis characterized by hemoptysis, chest pain and fever was absent in our patient. However, the combination of the radiological findings, elevated galactomannan antigen levels, histopathological presentation and a positive culture for A. fumigatus confirmed the diagnosis. Galactomannan is a constitutive antigen of the Aspergillus cell wall released during growth of hyphae that can be measured in the serum or bronchoalveolar lavage fluid. In invasive aspergillosis, the sensitivity and specificity of the method range from 61 to 71 and 89 to $93 \%$, respectively [14]. It is important to stress that the galactomannan assay exhibits cross-reaction with other agents, including Histoplasma. In addition, falsepositive results may occur in the presence of antibiotics, particularly piperacillin/tazobactam.

Although the first line of defense against inhaled conidia is represented by alveolar macrophages that trigger a neutrophil-mediated innate response, antigen-presenting cells play a key role in the activation of cellular adaptive immunity, in a process that involves CD4+ T-cell differentiation. Therefore, lymphopenia and, more importantly, CD4+ T-cell depletion, represent major predisposing host factors for invasive aspergillosis [13, 15]. As previously mentioned, selective CD4+ lymphopenia is a well-described adverse effect induced by TMZ. In a series of 97 patients with melanoma treated with TMZ $75 \mathrm{mg} / \mathrm{m}^{2}$ daily for 6 weeks, $60 \%$ of patients developed an absolute lymphocyte count $<800 / \mathrm{mm}^{2}$, with a 


\begin{tabular}{l|l}
\hline DOI: $10.1159 / 000354429$ & $\begin{array}{l}\text { C } 2013 \text { S. Karger AG, Basel } \\
\text { www.karger.com/cro }\end{array}$ \\
\hline
\end{tabular}

Munhoz et al.: Aspergillosis in a Patient Receiving Temozolomide for the Treatment of Glioblastoma

clear predominance of CD4+ T-cell depression [6]. In a recent publication by Grossman et al. [8], CD4+ T-cell counts of individuals with newly diagnosed high-grade gliomas receiving TMZ were prospectively evaluated. CD4+ T-cell counts lower than $300 / \mathrm{mm}^{3}$ occurred in $73 \%$ of patients, and survival was influenced by lymphocyte toxicity grades. Interestingly, nadir lymphocyte counts occurred approximately 2 months after initiating radiation and TMZ. In addition to lymphopenia, TMZ could also impair lymphocyte function through blockage of intracellular signaling pathways [16].

The possibility that prolonged use of corticosteroids could have contributed to the onset of the invasive infection must be taken into account; however, the immunosuppressive effect of TMZ has been described in subsets of populations for whom continuous use of corticosteroids is seldom recommended, including patients with melanoma and neuroendocrine tumors [6-8].

\section{Conclusion}

The case reported herein adds to the available evidence suggesting that patients receiving TMZ are at risk for aspergillosis, even though these events are rare. Since there is no recommendation for prophylaxis against opportunistic agents other than $P$. jiroveci, individuals in this situation should be closely monitored for infections, and even unspecific symptoms should prompt an immediate and thorough investigation.

\section{Disclosure Statement}

The authors have no potential conflict of interests to declare. No funding/financial support was received for this work.

\section{References}

1 Central Brain Tumor Registry of the United States: CBTRUS Statistical Report: Primary Brain and Central Nervous System Tumors Diagnosed in the United States in 2004-2007. Hinsdale, IL, CBTRUS, 2011. www.cbtrus.org.

2 Fisher JL, Schwartzbaum JA, Wrensch M, Wiemels JL: Epidemiology of brain tumors. Neurol Clin 2007;25:867-890.

3 Stupp R, Mason WP, van den Bent MJ, et al: Radiotherapy plus concomitant and adjuvant temozolomide for glioblastoma. N Engl J Med 2005;352:987-996.

4 Stupp R, Hegi ME, Mason WP, et al: Effects of radiotherapy with concomitant and adjuvant temozolomide versus radiotherapy alone on survival in glioblastoma in a randomised phase III study: 5-year analysis of the EORTC-NCIC trial. Lancet Oncol 2009;10:459-466.

5 Stupp R, Dietrich PY, Osternmann KS, et al: Promising survival for patients with newly diagnosed glioblastoma multiforme treated with concomitant radiation plus temozolomide, followed by adjuvant temozolomide. J Clin Oncol 2002;20:1375-1382.

6 Su YB, Sohn S, Krown SE, et al: Selective CD4+ lymphopenia in melanoma patients treated with temozolomide: a toxicity with therapeutic implications. J Clin Oncol 2004;22:610-616.

7 Schwarzberg AB, Stover EH, Sengupta T, et al: Selective lymphopenia and opportunistic infections in neuroendocrine tumor patients receiving temozolomide. Cancer Invest 2007;25:249-255.

8 Grossman SA, Ye X, Lesser G, et al: Immunosuppression in patients with high-grade gliomas treated with radiation and temozolomide. Clinical Cancer Res 2011;17:5473-5480.

9 Cohen MH, Johnson JR, Pazdur R: Food and Drug Administration drug approval summary: temozolomide plus radiation therapy for the treatment of newly diagnosed glioblastoma multiforme. Clin Cancer Res 2005;11:6767-6771.

10 Yaman E, Coskun U, Ozturk B, et al: Opportunistic cytomegalovirus infection in a patient receiving temozolomide for treatment of malignant glioma. J Clin Neurosci 2009;16:591-592. 


\section{Case Reports in Oncology}

\begin{tabular}{l|l}
\hline Case Rep Oncol 2013;6:410-415 & \\
\hline DOI: 10.1159/000354429 & $\begin{array}{l}\text { ○ 2013 S. Karger AG, Basel } \\
\text { www.karger.com/cro }\end{array}$ \\
\hline
\end{tabular}

Munhoz et al:: Aspergillosis in a Patient Receiving Temozolomide for the Treatment of Glioblastoma

11 Meije Y, Lizasoain M, Garcia-Reyne A, et al: Emergence of cytomegalovirus disease in patients receiving temozolomide: report of two cases and literature review. Clin Infect Dis 2010;50:e73-e76.

12 Limper AH, Knox KS, Sarosi GA, et al: An official American Thoracic Society statement: treatment of fungal infections in adult pulmonary and critical care patients. Am J Respir Crit Care Med 2011;183:96.

13 Segal BH: Aspergillosis. N Engl J Med 2009;360:1870-1884.

14 Pfeiffer CD, Fine JP, Safdar N: Diagnosis of invasive aspergillosis using a galactomannan assay: a metaanalysis. Clin Infect Dis 2006;42:1417.

15 Sterigipoulou T, Meletiadis J, Roilides E, et al: Host-dependent patterns of tissue injury in invasive pulmonary aspergillosis. Am J Clin Pathol 2007;127:349-355.

16 Wick W, Wick A, Schulz JB, et al: Prevention of irradiation-induced glioma cell invasion by temozolomide involves caspase 3 activity and cleavage of focal adhesion kinase. Cancer Res 2002;62:1915-1919.
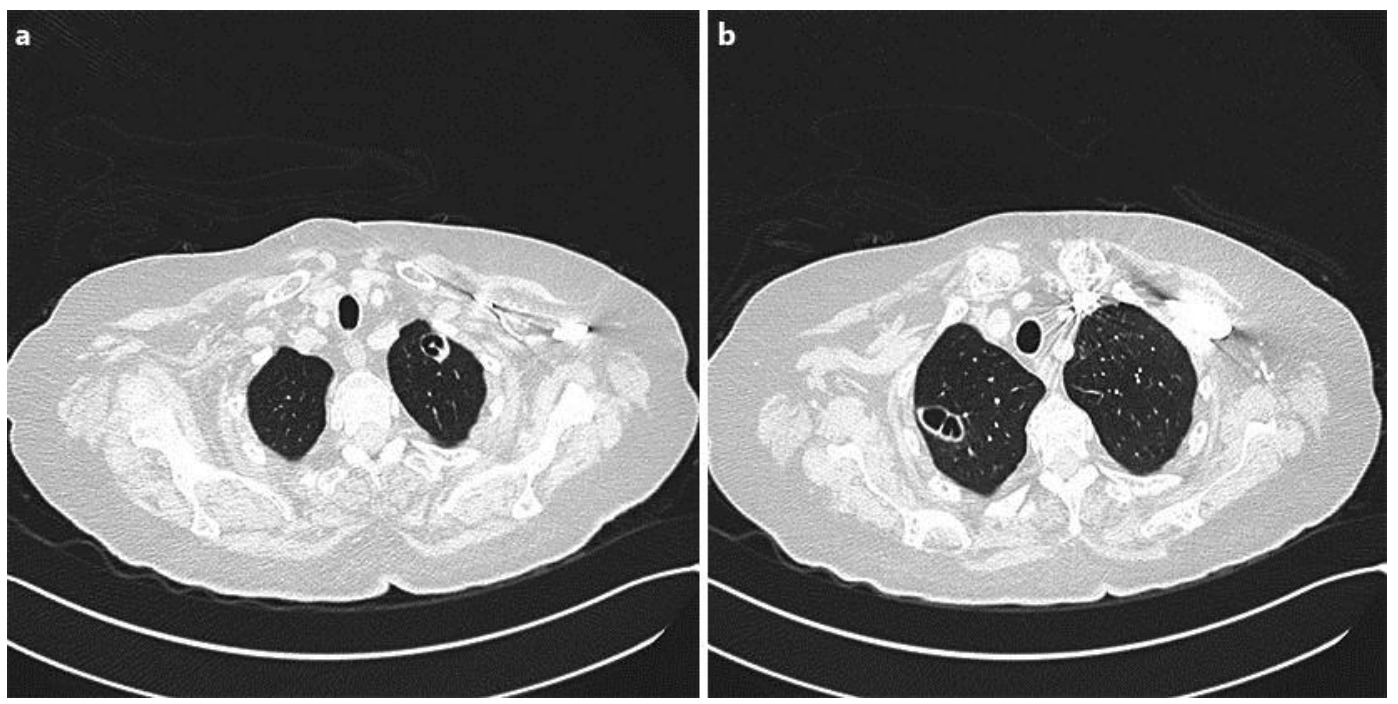

Fig. 1. a, b Chest CT scans performed at admission reveal findings compatible with a fungal infection.
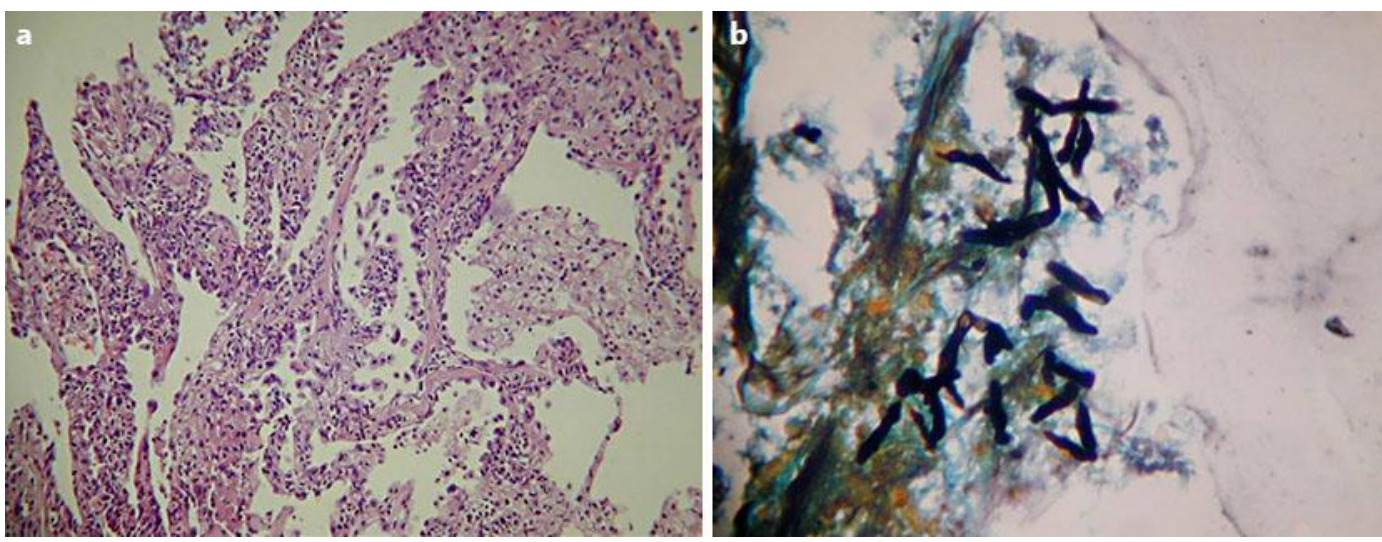

Fig. 2. a, b Lung biopsy. Hyphae could be visualized focally. Uniform, septate hyphae branching at $45^{\circ}$ angles confirm fungi consistent with Aspergillus. 


\section{Case Reports in Oncology}

\begin{tabular}{l|l}
\hline Case Rep Oncol 2013;6:410-415 \\
\hline DOI: 10.1159/000354429 & $\begin{array}{l}\text { @ 2013 S. Karger AG, Basel } \\
\text { www.karger.com/cro }\end{array}$ \\
\hline
\end{tabular}

Munhoz et al.: Aspergillosis in a Patient Receiving Temozolomide for the Treatment of Glioblastoma

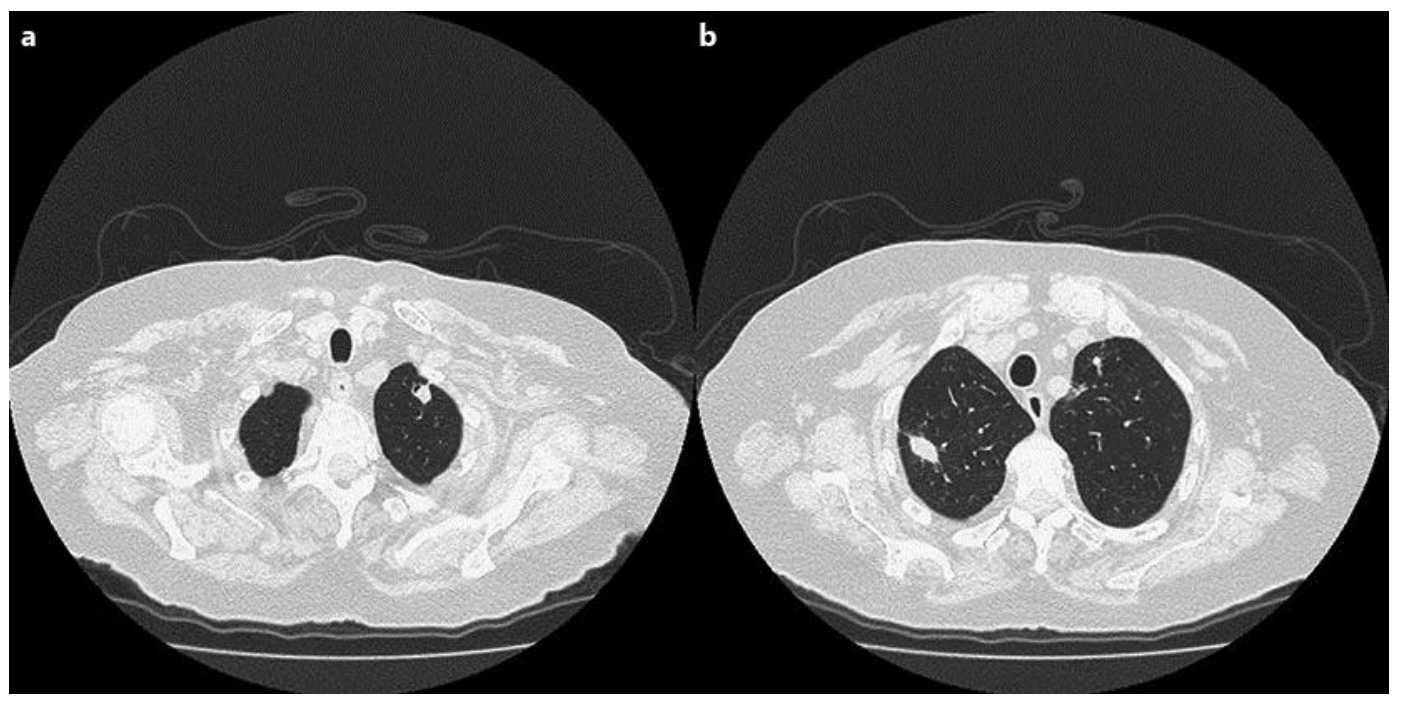

Fig. 3. a, b Control chest CT scans performed 30 days after admission show response to voriconazole. 